\title{
HIV PATIENT WITH MUCOUS MEMBRANE PEMPHIGOID: A CASE REPORT
}

\author{
Anand PratapSingh ${ }^{1}$, Chaitra T.R ${ }^{2}$, Ravishankar T.L ${ }^{3}$, Surendra Pratap \\ Singh $^{4}$, Ashok Kumar Mohapatra ${ }^{5}$
}

\begin{abstract}
BACKGROUND: Mucous Membrane Pemphigoid, a new denomination of cicatricial pemphigoid, encompasses a group of chronic subepithelial autoimmune blistering diseases that predominantly affect the oral cavity and the eyes (conjunctivitis and symblepharon).

CASE DETAILS: A rare case of Mucous Membrane Pemphigoid (MMP) in a human immunodeficiency virus (HIV) positive patient is discussed with clinicohistopathological presentation.

CONCLUSION: Since our patient was HIV-positive and had lesions restricted to the oral mucosa with ocular involvement, only topical and intralesional steroids were preferred as the first line of treatment. Systemic corticosteroid therapy raises a concern regarding immunosuppression.

KEYWORDS: HIV, MMP, Cicatricial pemphigoid.
\end{abstract}

\section{DOI: http://dx.doi.org/10.4314/ejhs.v24i2.12}

\section{INTRODUCTION}

MMP is autoimmune in nature and probably includes an autoantibody-induced, complement mediated sequestration of leukocytes with resultant cytokine and leukocyte enzyme release and detachment of the basal cells from the basement membrane zone, but there may also be complement-mediated cell lysis (1). MMP is characterized by junctional separation at the level of the basement membrane, causing a sub-basilar split.

MMP affects women twice as frequently as it affects men. It is also called cicatricialpemphigoid as it is attributed to scarring (2). HIV-related MMP is rarely reported in the medical literature. We report a case of MMP in a male patient who was also diagnosed as HIV positive during investigations for MMP and did not show any other oral manifestations.
We would also like to emphasize the need to investigate the immune status along with histopathology, immunofluorescence microscopy and Antibody Confirmation Tests for HIV, since HIV might have varying interactions with the immune system, which may lead to the development of autoimmune processes.

\section{CASE REPORT}

A forty-eight years old male patient reported with a chief complaint of painful ulcerations in his mouth and lip along with eye discomfort that had started before 10 days. He did not complain of the presence of any other systemic diseases or ongoing medical therapy, and did not report any changes in the skin or genital areas and related family history at the reporting date. The patient had normal physical and mental development with normal gait.

\footnotetext{
1Medical officer, CHC Nathnagar, Santkabir Nagar, Uttarpradesh

2Department of Pediatric Dentistry Kothiwal Dental College and Research Centre, Uttar Pradesh, India

${ }^{3}$ Department of Public Health Dentistry Kothiwal Dental College and Research Centre, Uttar Pradesh, India

${ }^{4}$ Department of Oral and Maxillofacial Surgery Kothiwal Dental College and Research Centre, Uttar Pradesh, India

${ }^{5}$ Dept. of Public Health Dentistry Indreprasta Dental College \& Hospital

Corresponding Author: Anand Pratap Singh, Email: anandsingh001@gmail.com
} 
Clinical assessment: Oral examination revealed large ulcerations formed after the rupture of bulla involving upper left buccal vestibule and mucosa surrounded by epithelial sloughing (Figure 1). Superficial, hemorrhagic crusted ulcerations on upper and lower labial mucosa and irregular ulceration involving the floor of the mouth (Figure 2) were well appreciable. A mild conjunctivitis with yellowish fibrinous slough and severe conjunctivitis were easily observed in the right and left eyes respectively (Figures 3). Further evaluation did not disclose any other lesions of buccal mucosa, perioral skin and genital areas. A mucosal biopsy was performed and the patient was recalled after five days for follow-up observation. However, on third day, the patient came with more extensive lesions on the left buccal mucosa, vestibule, upper and lower labial mucosa (Figure 4. It might be due to pressure on the normal mucosa applied during biopsy procedure (positive Nikolsky's sign). The possibility of a drug-induced bullous eruption was effectively excluded in this patient because he did not use any drugs.

The patient was treated with topical and intralesional corticosteroids $(0.1 \%$ triamcinolone acetonide and dexamethasone $4 \mathrm{mg} / \mathrm{ml}$ respectively). He was given instructions for oral hygiene care with Chlorhexidine Mouthwash $(0.2 \%)$ for five days and was referred to an ophthalmologist for conjunctival lesions.

A significant improvement was noticed in the lesion size and symptoms after ten days, and thereafter, the patient did not return for follow-up.

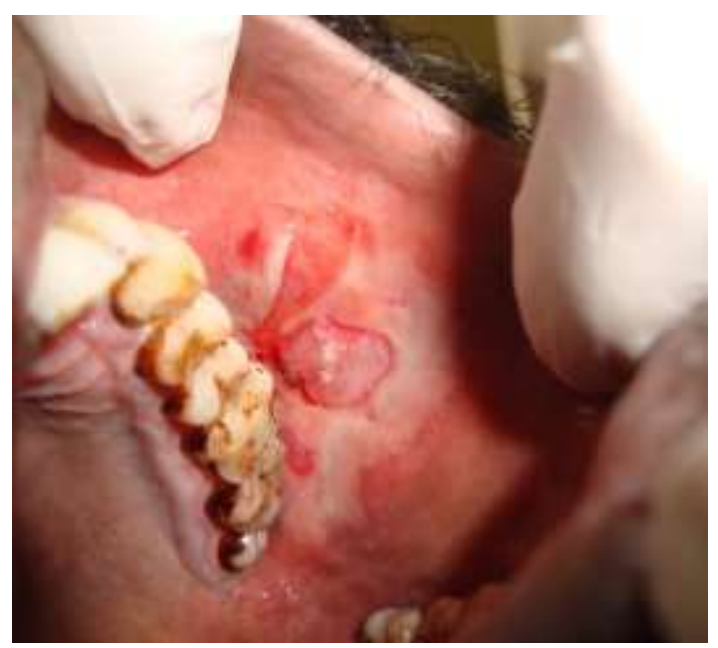

Fig1: Clinical picture showing the lesion

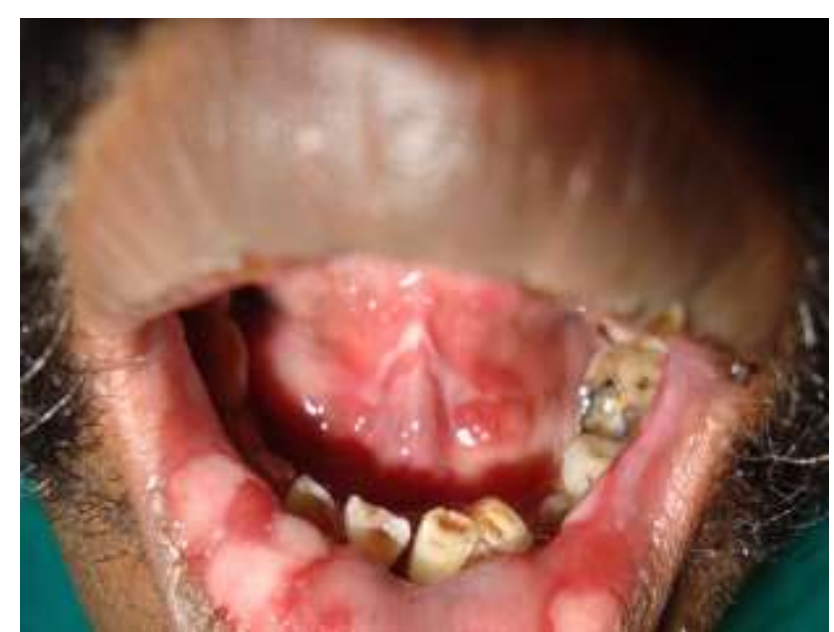

Figure 2: Showing lesion on floor of the mouth and lips

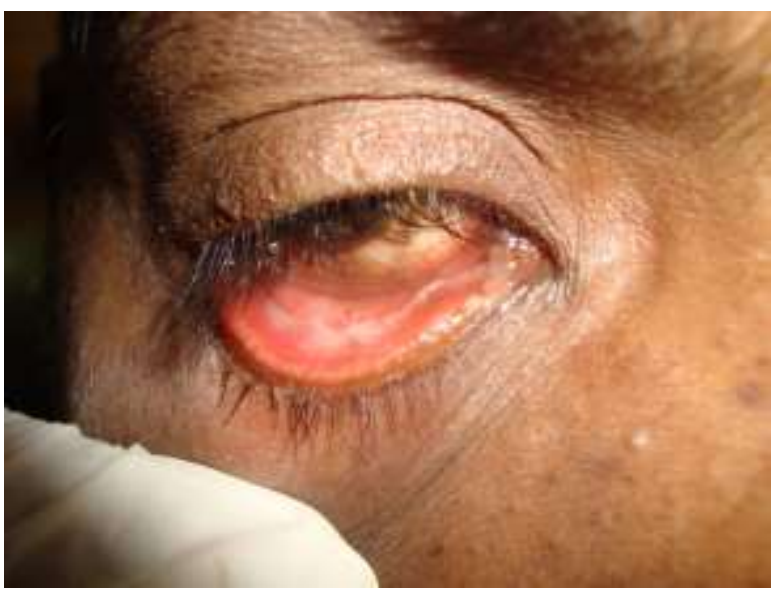

Figure 3: Showing eye involvement

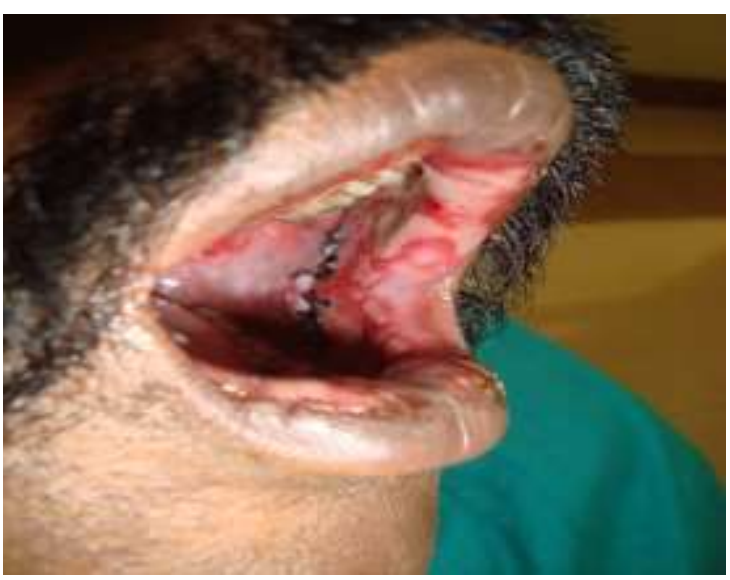

Figure 4: Extensive lesions after three days of biopsy 


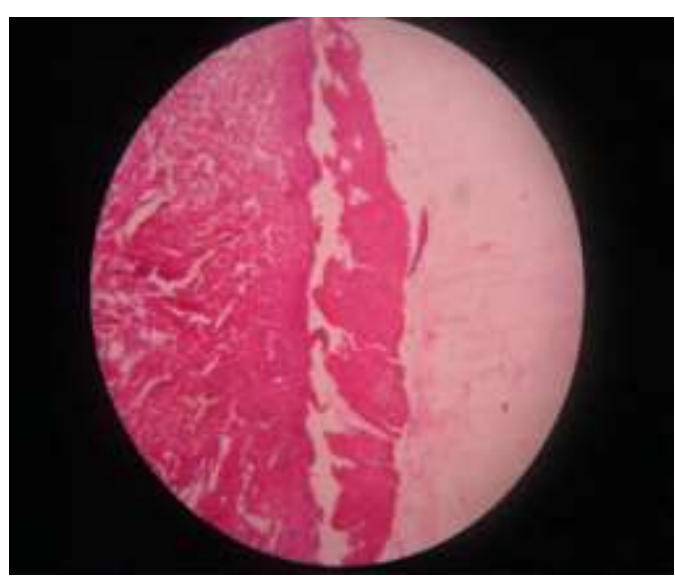

Figure 5: Photomicrograph of the lesion showing subepithelial cleft

Laboratory investigations: Routine blood investigations were found to be within normal range. Initially enzyme-linked immunosorbent assay (ELISA) and later Western blot were positive for HIV Antibody Confirmation Tests with 1180 CD4 cells /microlitre.

Immunofluorescence examination: Immunofluorescence testing could not be done because of unavailability of preservative transport media during biopsy.

Histopathological findings: Histopathology revealed the presence of Stratified squamous epithelium supported by fibrocellular connective tissue stroma and presence of subepithelial prominent areas of separation between the epithelium and connective tissue (Subepithelial cleft) with edematous connective tissue. Also dense infiltration of chronic inflammatory cells, predominantly the lymphocytes was seen (Figure5). There was also a presence of muscle tissue and adepocytes in the deeper portion of the tissue which was also infiltrated with inflammatory cells. Overall histopathological features were suggestive of mucous membrane pemphigoid.

Differential diagnosis: The following differential diagnosis of blistering diseases was considered before final diagnosis:

1. Erythema multiforme

2. Pemphigus vulgaris

3. Bullous pemphigoid

4. Erosive Lichen Planus

5. Behcet's syndrome

\section{DISCUSSION}

Mucous membrane pemphigoid has been known by many different names in the medical literature including benign mucous membrane pemphigoid, cicatricial (scarring) pemphigoid, and ocular cicatricialpemphigoid. A group of researchers in 2002(1) determined that mucous membrane pemphigoid was the best designation for this group of disorders. The term "benign" mucous membrane pemphigoid was deemed inappropriate because of the potential for serious complications in cases where the lesions were very severe. The term "cicatricial" pemphigoid excluded affected individuals who do not develop scarring. Sitespecific terms such as "ocular" cicatricialpemphigoid excluded individuals with multiple site involvement (1). Since in this patient characteristic lesions are restricted only to the mucosa and the eye, it was diagnosed as MMP (2, 3, 4).

Pathogenesis of MMP probably includes an autoantibody-induced, complement mediated sequestration of leukocytes with resultant cytokine and leukocyte enzyme release and detachment of the basal cells from the basement membrane zone, but there may also be complement-mediated cell lysis. MMP is characterized by junctional separation at the level of the basement membrane, which gives rise to a sub-basilar split (5), which was observed in the present histopathological section.

The association of immunodeficiency and autoimmunity in one patient is two sided clinical immune response. However, the reports of high frequency of auto antibodies in HIV-infected patients are increasing in the literature. Autoimmunity may develop during acute viral infection with normal to low CD4 counts (6), which may be a part of the nonspecific polyclonal B-cell stimulation secondary to interleukin (IL)-1 and IL-2 released by HIV-infected macrophages $(6,7)$. The frank loss of specific immunomodulatory CD4 $\mathrm{T}$ cells may allow the expansion of $\mathrm{B}$-cell clone, which is responsible for the autoantibody formation (8).

The actual pathogenesis and mechanism in development of mucous membrane pemphigoid secondary to HIV are still not confirmed. The possible mechanisms for autoimmune manifestations of HIV infection include the direct 
effect of HIV on endothelial, synovial, and other hematopoietic cells resulting in destruction of CD4 cells, increased cytotoxic cell activity and increased expression of auto-antigens (6). Molecular mimicry is one of the proposed mechanisms in the development of autoimmune disease. The exogenous infectious agent may have molecular similarity with a self-antigen and may therefore induce an autoimmune response $(7,8,9)$. Pemphigus is similar in its clinical presentation with MMP, but it produces acantholysis with cleavage of the spinous cell layer and the vesicle is intraepithelial, whereas in MMP as a result of the separation of connective tissue from the epithelium the vesicle is subepithelial (10). Keratinocyte necrosis, intracellular edema in prickle layer, acanthosis and a perivascular inflammatory cell infiltrate with a thin basement membrane adhered to the epithelium are characteristic histopathological features of Erythema multiforme (10).

In contrast to lichen planus, the inflammatory infiltrate is non-specific in nature comprising of lymphocytes, plasma cells neutrophils with few eosinophils. Patients with MMP rarely have circulating autoantibodies to the basement membrane zone components; thus, indirect immunofluorescence is usually not indicated as a diagnostic procedure.

Treatment of MMP is based on the severity of symptoms and the site involved $(1,2)$. Patients with mild localized lesions may often benefit from topical steroids such as beclomethasonedipropionate, betamethasone, clobetasol propionate, fluocinomide. Patients with more extensive lesions can be prescribed systemic steroids like prednisolone (1). Other treatment regimens, which are effective in certain resistant cases, are immunosuppressive agents such as azathioprine, cyclophosphamide, cyclosporine and dapsone. Sulphononamides and tetracyclines can also be implemented $(11,12)$. In HIV-infected individuals, oral corticosteroid therapy raises a concern regarding increased immunosuppression. Therefore, it should not be used in continuous treatment.

Since our patient was HIV-positive and had lesions restricted to the oral mucosa with ocular involvement, only topical and intralesional steroids were preferred as first-line treatment (1,
12) accompanied by referral of the patient to an ophthalmologist.

\section{REFERENCES}

1. Chan LS et al. The first International consensus on mucous membrane pemphigoid. Arch Dermatol, 2002; 138:370-9.

2. Alkan A, Gunhan O, Alkan A, Otan F. A clinical study of Oral Mucousmembrane Pemphigoid. J Int Med Res, 2003; 31(4): 3404.

3. Bozcurt FY, Celenligil H, Sungur A, Ruacan S. Gingival involvement in mucous membrane pemphigoid. Quintessence Int, 1998; 29: 438441.

4. Dayan S, Simmons R, Ahmed Contemporary issues in diagnosis of oral pemphigoidOralSurg Oral Med Oral Pathol Oral Radio Endod, 1999; 88: 424-30.

5. Scully C, Carrazzo M, Gandolofo S, Puialliu P, Montect R- Update on Mucous Membrane Pemphigoid. Oral Surg Oral Med Oral Patho Oral Radio Endo, 1999; 88; 56-68.

6. Goddard GZ, Shoenfeld Y. HIV and autoimmunity. Autoimmun Rev, 2002;1: 329337.

7. Etzioni A: Immune deficiency and autoimmunity. Autoimmun Rev 2003;2:364-9.

8. Demathe A, Arede LT, and Miyahara GI. Mucous membrane pemphigoid in HIV patient: a case report. Cases Journal, 2008; 1:345.

9. Scully C, Muzio LL: Oral mucosal diseases: mucous membrane pemphigoid. $\mathrm{Br} \mathrm{J}$ Oral MaxillofacSurg, 2008; 46:358-66.

10. Arisawa EAL, Almeida JD, Carvaiho RY, Cabral LAG. Clinicopathological analysis of oral mucous autoimmune disease: a 27-year study. Med Oral. Pathol. Cir Bucal. 2008 feb1;13 (2):E94-7.

11. Wojnarowska F, Kirtschig G, Highet AS, Venning VA, KhumaloNP.Guidelines for the management of bullous pemphigoid. $\mathrm{Br} j$ Dermatol, 2002 Aug; 147 (2): 214- 21.

12. Kirtschig G, Khumalo NP- Management of bullous pemphigoid:recommendations for immunomodulatory treatments. Am J ClinDermatol, 2004; 5(3):319-26. 